\title{
Comparação da composição bioquímica do líquido amniótico equino colhido em diferentes estágios gestacionais e no momento do parto ${ }^{1}$
}

\author{
Bruna De Vita ${ }^{2 *}$, Loreta L. Campos ${ }^{2}$, Amanda J. Listoni², Ian Martin², Regina K. \\ Takahira $^{3}$, Bruna R. Curcio ${ }^{4}$, Fernanda C. Landim-Alvarenga ${ }^{2}$ e Nereu C. Prestes ${ }^{2}$
}

ABSTRACT.- De Vita B., Campos L.L., Listoni A.J., Martin I., Takahira R.K., Curcio B.R., Lan-
dim-Alvarenga F.C. \& Prestes N.C. 2014. [Comparison of the biochemical composition
of equine amniotic fluid collected in different gestational phases and at delivery.]
Comparação da composição bioquímica do líquido amniótico equino colhido em diferentes
estágios gestacionais e no momento do parto. Pesquisa Veterinária Brasileira 34(6):582-
588. Departamento de Reprodução Animal e Radiologia Veterinária, Faculdade de Medicina
Veterinária e Zootecnia, Universidade Estadual Paulista, Distrito de Rubião Júnior s/n, Bo-
tucatu, SP 18618-970, Brazil. E-mail: bruddev@gmail.com

The viability and fetal maturity can be estimated by biochemical evaluation of the fetal fluids of several species; however the biochemical composition of amniotic fluid during pregnancy is not fully defined for equine. The aim of this study was to establish and compare the biochemical profile of amniotic fluid in different moments of pregnancy and at delivery, in order to better explain the peculiarities of the physiology of pregnancy in mares. The values founded for $\mathrm{pH}$, osmolarity, glucose, urea, creatinine, gamma-GT, Sodium, potassium, chloride and total protein were evaluated in amniotic fluid collected from 122 mares comparing the results between the initial-third (IT), mid-third (MT) and latter-third (FT) of gestation and at delivery (D). The gestational period samples were collected at slaughterhouses. Gestational ages (IG), in days, were defined following the regression formula suggested by Naves et al. (2008) by measuring the craniocaudal (CC) distance of the fetuses. Commercial kits for biochemical evaluation were used. Due to the presence of varying degrees of asymmetry and deviations from a standard Gaussian distribution, the Kruskal-Wallis test was used to compare the median of each response variable among the study groups. When there has been significant evidence that at least one of the medians differed from the others, the Wilcoxon test was used to perform multiple comparisons between groups. The Bonferroni method was used to adjust the resulting $p$-value for multiple comparisons. Statistical analysis was performed using PROC Npar1way and statistical significance was defined as $\mathrm{p}<0.05$. The $\mathrm{pH}$ and osmolarity of equine amniotic fluid did not change significantly during the stages of pregnancy and at delivery. The values found for glucose were significantly lower during late pregnancy and at delivery. Concentrations of urea tended to be statistically different in at least one of the groups. A significant increase in creatinine concentrations was observed during the initial-third, medium-third and the final-third of pregnancy and the value found at delivery remained equal to final-third. Values for Gamma GT differed only between FT and D groups and more studies should be conducted about its role in the amniotic fluid of domestic species. For the sodium and chloride

\footnotetext{
${ }^{1}$ Recebido em 22 de setembro de 2013.

Aceito para publicação em 23 de fevereiro de 2014.

${ }^{2}$ Departamento de Reprodução Animal e Radiologia Veterinária, Faculdade de Medicina Veterinária e Zootecnia (FMVZ), Universidade Estadual Paulista (Unesp), Distrito de Rubião Júnior s/n, Botucatu, SP 18618-970, Brasil. *Autor para correspondência: bruddev@gmail.com
}

\footnotetext{
${ }^{3}$ Departamento de Clínica Veterinária, Laboratório Clínico, Faculdade de Medicina Veterinária e Zootecnia, Universidade Estadual Paulista (Unesp), Botucatu, SP 18618-970.

${ }^{4}$ Departamento de Clínicas Veterinárias, Faculdade de Medicina Veterinária (FMV), Universidade Federal de Pelotas (UFPel), Rua Gomes Carneiro 1, Centro, Pelotas, RS 96010-610, Brasil.
} 
ions, they were not significantly different between the studied stages, and the potassium ion was significantly different only between FT and D reflecting the maintenance of electrolyte balance of the amniotic fluid during equine pregnancy. Total protein concentrations were different between groups, but all the groups showed low concentrations. We conclude that the values of the parameters studied varied according with the fetal development and can be used for the evaluation of fetal viability and maturity in the future, but the scarce number of studies in this area and the difference between the results found in literature demonstrate the need of more works to establish the biochemical profile of equine amniotic fluid during pregnancy and at delivery.

INDEX TERMS: Equine, amniotic fluid, biochemical evaluation, fetal viability.

RESUMO.- A viabilidade e maturidade fetais podem ser estimadas através da avaliação dos fluídos fetais em muitas espécies, no entanto a composição bioquímica do líquido amniótico durante a gestação ainda não está bem definida para a espécie equina. 0 objetivo deste estudo foi estabelecer e comparar o perfil bioquímico do liquido amniótico em diferentes momentos da gestação e no momento do parto para um melhor entendimento da fisiologia da gestação de equinos. Foram avaliados valores encontrados para $\mathrm{pH}$, osmolaridade, glicose, uréia, creatinina, gamma-GT, sódio, potássio, cloretos e proteína total no liquido amniótico colhido de 122 éguas comparando-se os resultados encontrados entre as colheitas em terço inicial (TI), médio (TM) e final (TF) da gestação e no momento do parto (MP). As amostras colhidas nos períodos gestacionais foram realizadas em frigoríficos abatedouros. As idades gestacionais (IG), em dias, foram definidas através de fórmula de regressão sugerida por Naves et al. (2008), utilizando-se a mensuração crânio-caudal dos fetos. Devido a presença de graus variados de assimetria e desvios de um padrão Gaussiano de distribuição, o teste de Kruskal-Wallis foi usado para comparar a mediana de cada variável-resposta entre os grupos de estudo. Quando houve evidência significante de que pelo menos uma das medianas era diferente das outras, o teste de Wilcoxon foi usado para realizar comparações múltiplas entre os grupos. 0 método de Bonferroni foi usado para ajustar o valor-P resultante das comparações múltiplas. A análise estatística foi realizada com o procedimento PROC NPAR1WAY e significância estatística foi definida como $\mathrm{p}<0,05$.pH e osmolaridade do líquido amniótico equino não sofreram alterações significantes entre os terços gestacionais e no momento do parto. Os valores encontrados para glicose foram significativamente menores nas amostras do grupo TF e MP. As concentrações de uréia tenderam ser estatisticamente diferente em pelo menos um dos grupos estudados e foi observado um padrão crescente nas concentrações de creatinina durante as fases gestacionais, enquanto que a concentração encontrada no grupo MP foi igual a concentração encontrada no grupo TF. Valores para gamma GT diferiram apenas entre os grupos TF e MP e mais estudos devem ser conduzidos sobre sua função no liquido amniótico das espécies domésticas. Os valores de sódio e cloretos não diferiram significativamente entre os grupos estudados e as concentrações de potássio diferiram apenas entre os grupos TF e MP refletindo a manutenção do equilíbrio eletrolítico do líquido amniótico equino durante a gestação. As concentrações de proteínas totais variaram de maneira heterogênea entre os grupos, porém todos estes demonstraram baixas concentrações. Concluímos que a composição bioquímica do liquido amniótico equino varia de acordo com o desenvolvimento fetal e pode ser utilizada como mensuração da viabilidade e maturidade do feto no futuro, no entanto devido ao pequeno número de estudos conduzidos e diferenças encontradas em seus resultados, outros estudos devem ser realizados para a melhor definição do perfil bioquímico do líquido amniótico equino durante as fases gestacionais e no momento do parto.

TERMOS DE INDEXAÇÃO: Equino, líquido amniótico, avaliação bioquímica, viabilidade fetal.

\section{INTRODUÇÃO}

Em medicina humana, a amniocentese de mulheres e a posterior análise do líquido amniótico são utilizadas rotineiramente para detectar principalmente doenças congênitas, defeitos do tubo neural, idade gestacional e maturidade pulmonar fetal. 0 exame é indicado para mulheres acima de 35 anos devido à maior probabilidade de anormalidades cromossômicas fetais (Campana et al. 2003).

Segundo Vaala \& House (2002), enfermidades e a viabilidade do feto humano podem ser precisamente determinadas através do exame ultrassonográfico do feto no interior do útero associado à composição bioquímica do líquido amniótico obtida por amniocentese, guiada por ultrassonografia.

Em medicina veterinária, os primeiros estudos feitos para análise da fisiologia e comportamento bioquímico do líquido amniótico, foram realizados em ovinos. Alexander et al. (1958) procederam amniocentese em ovelhas com o objetivo de determinar a variação na composição do líquido fetal e urina durante a gestação. Promoveram punções no âmnion, alantóide e bexiga dos 45 aos 142 dias da prenhez, afim de determinar a pressão osmótica, nitrogênio total e não-proteico, amino-ácidos, uréia, sulfato, fosfato total e inorgânico, creatinina, ácido úrico, $\mathrm{Na}, \mathrm{K}, \mathrm{Cl}$, pH e osmolaridade.

Prestes et al. (2001) avaliaram os componentes do fluído amniótico de ovelhas aos 70, 100 e 145 dias de gestação sendo que, os valores obtidos para os três estágios gestacionais respectivamente foram: $\mathrm{pH}(8,36 ; 7,34 \mathrm{e}$ $7,37)$, glicose $(16,06 ; 8,58$ e $3,79 \mathrm{~g} / \mathrm{dL})$, uréia $(42,68 ; 33,53$; $25,49 \mathrm{mg} / \mathrm{dL})$, creatinina $(0,85 ; 5,04 ; 11,25 \mathrm{~g} / \mathrm{dL})$, sódio $(146,60 ; 129,42$ e $103,8 \mathrm{mmol} / \mathrm{L})$, potássio $(9,79 ; 6,15$; e $8,65 \mathrm{mmol} / \mathrm{L})$, cloreto $(96,59 ; 85,28$ e $65,35 \mathrm{mmol} / \mathrm{L})$ e 
proteína total $(0,14 ; 0,23$ e $0,24 \mathrm{~g} / \mathrm{dL})$. Os autores concluíram que os valores absolutos do $\mathrm{pH}$, glicose, uréia, sódio e cloretos decresceram durante a gestação, apesar de não terem diferido significativamente e que os valores absolutos de creatinina e proteína total aumentaram aos 100 e 145 de gestação em comparação aos 70 dias, embora os valores dessas proteínas tenham sido similares entre os dias 100 e 145.

Martins et al., (2004) promoveram a análise comparativa dos componentes bioquímicos no líquido amniótico de fetos e soro de cadelas. Constataram acidose em 14,3\% das mães, enquanto $85,7 \%$ apresentavam-se em alcalose. Descreveram ainda, que a uréia foi o parâmetro em que se observou maior correlação positiva entre os valores obtidos das mães com os valores médios dos filhotes. A concentração de creatinina não apresentou nenhum tipo de correlação, quanto à dosagem de cloreto, mães com baixo teor também exibiram filhotes com baixo teor no líquido amniótico e vice-versa. A concentração de gama GT foi superior no líquido amniótico. Resultado inverso foi observado para glicose sendo que o alto teor do açúcar no soro refletiu-se no líquido amniótico. Sódio, potássio, cálcio e fósforo tiveram valores semelhantes em ambos os compartimentos. A proteína total no líquido amniótico foi detectada em quantidades ínfimas, mesmo para os produtos cujas mães apresentavam teores dentro do padrão fisiológico. Para a variável magnésio observou-se o predomínio no líquido amniótico.

Barreto et al. (2006), analisaram os valores de $\mathrm{pH}$ e bioquímicos determinados com o auxílio de "kits" comerciais para Proteína Total (PT), Uréia (Ure), Creatinina (Cre), Cloretos Totais (Cl) e Glicose (Gli) nos fluidos amniótico e alantoideano de cadelas entre 30 a 40 dias de gestação e observaram que dos constituintes propostos para análise, os valores de PT encontrados para o fluido amniótico $(35,47 \mathrm{mg} / \mathrm{dL})$ foram significativamente inferiores ao do alantoideano $(56,55 \mathrm{mg} / \mathrm{dL})$. Este constituinte apresentou resultados semelhantes em bufalinos (Souza et al. 1999). Para Kjeldsberg \& Kninght (1993) e Moore \& Persaud (2000) as proteínas são os elementos mais abundantes e que sofrem maior influência da fisiologia fetal.

$\mathrm{Na}$ avaliação dos elementos glicose, uréia, creatinina, íon cloro e pH no experimento de Barreto et al. (2006) não foram observadas diferenças podendo refletir a sincronia dos fluidos na manutenção do equilíbrio eletrolítico nestes dois compartimentos. As proteínas totais foram correlacionadas positivamente com a uréia e a creatinina do fluido amniótico, o que não ocorreu no fluido alantoideano. Esta correlação pode refletir o início da funcionalidade do sistema renal (Çagdas et al. 2000).

Para a espécie equina, não está totalmente definida a composição do líquido amniótico no decorrer da gestação. Os trabalhos nesta espécie são escassos. Portanto, ainda não existem métodos capazes de determinar com exatidão o grau de maturidade de alguns órgãos. Assim, ainda é difícil estabelecer, juntamente com outros métodos aplicados em perinatologia, a idade gestacional do concepto equino e, por conseguinte, estabelecer o nível de estresse que esse produto irá enfrentar na hora do parto (Vaala, 2002).
Williams et al. (1993), relataram que nos equinos, a vesícula amniótica flutua livre na cavidade alantoideana ligada somente pelo segmento umbilical. A cavidade alantoideana armazena produtos de excreção renal que passam via úraco. 0 líquido amniótico permite o crescimento e movimentos do feto e acumula células epiteliais e secreção nasofaringeal. A amniocentese foi realizada em 10 éguas ao final da gestação $(323,8 \pm 10,2$ dias $)$ com o objetivo primordial de mensurar os valores de fosfatidilglicerol e lecitina-esfingomielina que em medicina humana, são utilizados para determinar a maturidade pulmonar. Avaliaram também as concentrações de cortisol e creatinina. Para cortisol (mmol/l), aos 338, 360 e 180 dias da gestação, os valores encontrados foram 66, 13 e 1, enquanto para creatinina (mg/dL) no mesmo período, os valores foram de 1140,4 , 2254,2 e 203,3, respectivamente. 0 líquido foi coletado de ambas as cavidades e imediatamente analisados em laboratório. Comparado ao líquido amniótico, os valores encontrados no líquido alantoideano foram significativamente mais altos para, densidade específica, Bilirrubina total, Gama Glutamil Transferase, Fósforo, Proteína Total e Globulina, e foram mais baixos para $\mathrm{Na}^{+}, \mathrm{Cl}^{-}$e Fosfatase Alcalina. Concluíram, que o conhecimento do comportamento dos líquidos fetais é fundamental para se aferir a viabilidade e bem estar do feto.

Kochhar et al. (1997), avaliaram os líquidos fetais de 22 éguas coletados no momento do parto por centese. Metade destas apresentaram distocias no parto. Nestas éguas, os valores de proteína total encontrados foram mais baixos e os valores encontrados para uréia, creatinina e glicose foram mais altos do que os valores encontrados no grupo de éguas que não apresentaram distocias. 0 valor de sódio no líquido alantoideano foi bem menor do que o encontrado no líquido amniótico. Os valores obtidos da dosagem dos íons potássio e cálcio mostraram que os mesmos apresentaram-se maiores no fluido alantoideano. No entanto, os autores destacam que a intensidade e o tipo de trabalho de parto podem interferir na composição dos fluidos fetais nesta espécie.

Zanella (2008) estudou a composição bioquímica dos fluidos fetais nos diferentes estágios da gestação de éguas. $\mathrm{O}$ autor observou que a fosfatase alcalina, proteína total, íons cálcio, cloreto e sódio são mais elevados no terço médio da gestação. A concentração de uréia é maior fase inicial, a concentração da creatinina se eleva durante a gestação e a concentração do íon potássio não se eleva durante as fases gestacionais. Relatou também, que na comparação das médias das concentrações do líquido amniótico e líquido alantoideano, as concentações da fosfatase alcalina, proteína total, íons cloreto e sódio são maiores no líquido amniótico durante as três fases da gestação. As concentrações da glicose e da uréia não apresentam diferenças entre os dois compartimentos durante o período gestacional e que, as concentrações dos íons cálcio e potássio são maiores no líquido alantoideano.

A viabilidade fetal na espécie eqüina pode ser avaliada através de dosagens dos parâmetros bioquímicos, hormonais e exames ultrassonográficos dos líquidos fetais e placenta. Mas para tanto, se faz necessário mais estudos para 
identificar a composição dos líquidos fetais em cada fase da gestação (Jonker, 2004). Sendo assim, este trabalho teve como objetivo avaliar o perfil bioquímico do líquido amniótico equino colhido no primeiro, segundo e terceiro terço gestacionais e compará-los para um melhor entendimento da fisiologia da gestação equina e futura utilização destes parâmetros como ferramenta para avaliação da maturidade e viabilidade fetal equina.

\section{MATERIAL E MÉTODOS}

\section{Colheita e armazenamento das amostras}

Amostras de aproximadamente $15 \mathrm{~mL}$ de líquido amniótico foram colhidas de úteros gravídicos por aspiração com seringa agulhada, em frigoríficos comerciais, de éguas sem raça definida e no momento do parto, quando do início da insinuação do feto no canal de parição, de éguas da raça puro sangue inglês. As amostras foram armazenadas em tubos de centrífuga de $15 \mathrm{~mL}$ e mantidas sob $-20^{\circ} \mathrm{C}$ em freezer até o momento das análises.

Os fetos das fases gestacionais tiveram a distância encéfalo coccígea mensuradas para o estabelecimento da idade fetal segundo a equação sugerida por Neves et al (2008): $\mathrm{IG}=22,623+$ $4,2528 \mathrm{CC}-0,0124 \mathrm{CC}^{2}\left(\mathrm{r}_{2}=0,98\right)$ e a formação dos grupos : Terço Inicial (TI entre 39 -112 dias), Terço Médio (TM - entre 113 e 224 dias), Terço Final (TF - além de 225 dias) e Momento do Parto (MP), demonstrados no quadro 1.

\section{Processamento das amostras}

As amostras foram descongeladas em recipiente contendo água e gelo em ambiente refrigerado com temperatura controlada. Após a descongelação foi realizada uma centrifugação refrigerada a $5^{\circ} \mathrm{C}$ a $2500 \mathrm{rpm}$ durante 20 minutos para a formação do pellet de células e separação do excesso de muco. Uma alíquota de $3 \mathrm{~mL}$ do sobrenadante foi separada para avaliação do $\mathrm{pH}$ e da osmolaridade; outra alíquota, de $1 \mathrm{~mL}$, foi retirada para avaliação da glicose, ureia, creatinina, cloretos, gama GT, sódio, potássio e proteína total (Kits Celm ou Katal compatíveis com o equipamento FC-280.

\section{Análise estatística}

Inicialmente a distribuição das variáveis-resposta foi analisada com um dos critérios para escolha do método analítico. Devido à presença de graus variados de assimetria e desvios de um padrão Gaussiano de distribuição, o teste de Kruskal-Wallis (Pagano \& Gauvreau, 2000) foi usado para comparar a mediana de cada variável-resposta entre os grupos de estudo. Quando houve evidência significante de que pelo menos uma das medianas era diferente das outras, o teste de Wilcoxon (Pagano \& Gauvreau 2000) foi utilizado para realizar comparações múltiplas entre os grupos. 0 método de Bonferroni (Kleinbaum et al. 2007) foi usado para ajustar o valor-P resultante das comparações múltiplas. A análise estatística foi realizada com o procedimento PROC NPAR1WAY (SAS Institute 2009) e a significância estatística foi definida como $P<0.05$.

\section{RESULTADOS}

Os resultados encontrados para $\mathrm{pH}$, osmolaridade (mOsm/L), glicose $(\mathrm{mg} / \mathrm{dL})$, uréia $(\mathrm{mg} / \mathrm{dL})$, creatinina $(\mathrm{mg} / \mathrm{dL})$, gamma-GT (IU/L), sódium (mEq/L), potássio $(\mathrm{mEq} / \mathrm{L})$, cloretos $(\mathrm{mEq} / \mathrm{L})$ e proteína total $(\mathrm{mg} / \mathrm{dL})$ no líquido amniótico equino colhido nos diferentes momentos estudados estão demonstrados nos Quadros 2 e 4-11, respectivamente, e na Figura 1.
Quadro 1. Quantidade de amostras de líquido amniótico equino colhidas e separadas entre os grupos Terço Inicial (TI), Terço Médio (TM), Terço Final (TF) e Momento do Parto (MP) de acordo com a mensuração encéfalo-coccígea dos fetos

\begin{tabular}{lc} 
Grupos & Quantidade de amostras \\
\hline TI & 9 \\
TM & 28 \\
TF & 30 \\
MP & 55 \\
Total & 122
\end{tabular}

Quadro 2. Valores de pH em unidades de pH, quantidades de amostras ( $\mathrm{N}$ ), média, desvio padrão, mediana e erro padrão encontrados em amostras do líquido amniótico equino colhido no Terço Inicial, Terço Médio e Terço Final da gestação e no momento do parto

\begin{tabular}{lccccc}
\hline Grupo & N & Média & Desvio Padrão & Mediana & Erro Padrão \\
\hline TI & 9 & 7,81 & 1,00 & 8,17 & 0,33 \\
TM & 28 & 8,10 & 0,66 & 8,15 & 0,13 \\
TF & 30 & 7,86 & 0,47 & 7,77 & 0,09 \\
MP & 55 & 8,00 & 0,50 & 7,84 & 0,07
\end{tabular}

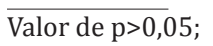

Quadro 3. Valores de osmolaridade em m0sm/L, quantidades de amostras (N), média, desvio padrão, mediana e erro padrão encontrados em amostras do líquido amniótico equino colhido no Terço Inicial, Terço Médio e Terço Final da gestação e no momento do parto

\begin{tabular}{lccccc}
\hline Grupo & N & Média & Desvio Padrão & Mediana & Erro Padrão \\
\hline TI & 9 & 255,50 & 141,70 & 296,50 & 50,10 \\
TM & 28 & 293,56 & 134,99 & 250,00 & 25,98 \\
TF & 30 & 334,63 & 112,55 & 320,00 & 20,55 \\
MP & 55 & 298,91 & 115,26 & 269,00 & 15,83
\end{tabular}

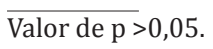

Quadro 4. Valores de glicose em $\mathrm{mg} / \mathrm{dL}$, quantidades de amostras $(\mathrm{N})$, média, desvio padrão, mediana e erro padrão encontrados em amostras do líquido amniótico equino colhido no Terço Inicial, Terço Médio e Terço Final da gestação e no momento do parto

\begin{tabular}{lccccc}
\hline Grupo & N & Média & Desvio Padrão & Mediana & Erro Padrão \\
\hline TI & 9 & 12,44 & 13,36 & $6,00^{\mathrm{a}}$ & 4,45 \\
TM & 28 & 11,86 & 10,44 & $8,50^{\mathrm{a}}$ & 1,97 \\
TF & 30 & 2,40 & 2,82 & $1,00^{\mathrm{b}}$ & 0,52 \\
MP & 55 & 3,91 & 13,97 & $1,00^{\mathrm{b}}$ & 1,88
\end{tabular}

Letras diferentes na mesma coluna indicam haver diferença significativa. Valor de $\mathrm{p}<0,008$.

Quadro 5. Valores de uréia em $\mathrm{mg} / \mathrm{dL}$, quantidades de amostras $(\mathrm{N})$, média, desvio padrão, mediana e erro padrão encontrados em amostras do líquido amniótico equino colhido no Terço Inicial, Terço Médio e Terço Final da gestação e no momento do parto

\begin{tabular}{lccccc}
\hline Grupo & N & Média & Desvio Padrão & Mediana & Erro Padrão \\
\hline TI & 9 & 32,88 & 12,36 & 34,10 & 4,12 \\
TM & 28 & 34,22 & 40,02 & 20,80 & 7,56 \\
TF & 30 & 33,14 & 19,70 & 26,25 & 3,60 \\
MP & 55 & 36,64 & 16,35 & 33,20 & 2,20
\end{tabular}

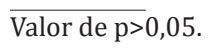


Quadro 6. Valores de uréia em $\mathrm{mg} / \mathrm{dL}$, quantidades de amostras (N), média, desvio padrão, mediana e erro padrão encontrados em amostras do líquido amniótico equino colhido no Terço Inicial, Terço Médio e Terço Final da gestação e no momento do parto

\begin{tabular}{lccccc}
\hline Grupo & $\mathrm{N}$ & Média & Desvio Padrão & Mediana & Erro Padrão \\
\hline TI & 9 & 0,42 & 0,33 & $0,30^{\mathrm{a}}$ & 0,11 \\
TM & 28 & 1,31 & 1,10 & $0,80^{\mathrm{b}}$ & 0,21 \\
TF & 30 & 6,57 & 5,08 & $4,95^{\mathrm{c}}$ & 0,93 \\
MP & 55 & 5,33 & 3,40 & $4,30^{\mathrm{c}}$ & 0,46
\end{tabular}

Letras diferentes na mesma coluna indicam haver diferença significativa. Valor de $\mathrm{p}<0,008$;

Quadro 7. Valores de GGT em UI/L, quantidades de amostras (N), média, desvio padrão, mediana e erro padrão encontrados em amostras do líquido amniótico equino colhido no Terço Inicial, Terço Médio e Terço Final da gestação e no momento do parto

\begin{tabular}{lccccc}
\hline Grupo & N & Média & Desvio Padrão & Mediana & Erro Padrão \\
\hline TI & 9 & 6,80 & 11,21 & $3,00^{\mathrm{a}}$ & 3,74 \\
TM & 28 & 5,69 & 3,65 & $5,65^{\mathrm{a}}$ & 0,69 \\
TF & 30 & 7,10 & 3,78 & $6,20^{\text {ab }}$ & 0,69 \\
MP & 55 & 4,51 & 2,87 & $3,70^{\text {ac }}$ & 0,39
\end{tabular}

Letras diferentes na mesma coluna indicam haver diferença significativa. Valor de $\mathrm{p}<0,008$.

Quadro 8: Valores do íon Na em $\mathrm{mEq} / \mathrm{L}$, quantidades de amostras $(\mathrm{N})$, média, desvio padrão, mediana e erro padrão encontrados em amostras do líquido amniótico equino colhido no Terço Inicial, Terço Médio e Terço Final da gestação e no momento do parto

\begin{tabular}{lccccc}
\hline Grupo & N & Média & Desvio Padrão & Mediana & Erro Padrão \\
\hline TI & 9 & 51,67 & 49,30 & 45,00 & 16,43 \\
TM & 28 & 66,75 & 43,68 & 51,50 & 8,26 \\
TF & 30 & 75,73 & 40,36 & 63,00 & 7,37 \\
MP & 55 & 61,40 & 22,63 & 55,00 & 3,05
\end{tabular}

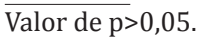

Quadro 9. Valores do íon K em mEq/L, quantidades de amostras ( $\mathrm{N})$, média, desvio padrão, mediana e erro padrão encontrados em amostras do líquido amniótico equino colhido no Terço Inicial, Terço Médio e Terço Final da gestação e no momento do parto

\begin{tabular}{lccccc}
\hline Grupo & $\mathrm{N}$ & Média & Desvio Padrão & Mediana & Erro Padrão \\
\hline TI & 9 & 2,40 & 1,56 & $1,80^{\mathrm{a}}$ & 0,52 \\
TM & 28 & 2,76 & 1,87 & $1,95^{\mathrm{a}}$ & 0,35 \\
TF & 30 & 4,04 & 2,90 & $3,15^{\text {ab }}$ & 0,53 \\
MP & 55 & 2,11 & 0,94 & $1,90^{\text {ac }}$ & 0,13
\end{tabular}

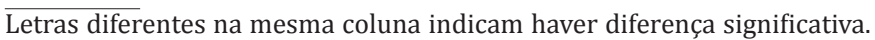
Valor de $\mathrm{p}<0,008$.

\section{DISCUSSÃO}

A composição do líquido amniótico no decorrer da gestação não está totalmente definida para a espécie equina. Os trabalhos nesta espécie são escassos, portanto ainda não existem métodos capazes de determinar com exatidão o grau de maturidade de alguns órgãos. Sendo assim, ainda é difícil estabelecer, juntamente com outros métodos aplicados em perinatologia, a idade gestacional do concepto equino
Quadro 10. Valores do íon Cl em mEq/L, quantidades de amostras $(\mathrm{N})$, média, desvio padrão, mediana e erro padrão encontrados em amostras do líquido amniótico equino colhido no Terço Inicial, Terço Médio e Terço Final da gestação e no momento do parto

\begin{tabular}{lccccc}
\hline Grupo & N & Média & Desvio Padrão & Mediana & Erro Padrão \\
\hline TI & 9 & 60,56 & 56,95 & 58,00 & 18,98 \\
TM & 28 & 84,32 & 60,65 & 67,00 & 11,46 \\
TF & 30 & 89,37 & 37,91 & 83,50 & 6,92 \\
MP & 55 & 73,93 & 28,38 & 68,00 & 3,83
\end{tabular}

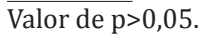

Quadro 11. Valores de proteína total em $\mathrm{mg} / \mathrm{dL}$, quantidades de amostras $(\mathrm{N})$, média, desvio padrão, mediana e erro padrão encontrados em amostras do líquido amniótico equino colhido no Terço Inicial, Terço Médio e Terço Final da gestação e no momento do parto

\begin{tabular}{lccccc}
\hline Grupo & N & Média & Desvio Padrão & Mediana & Erro Padrão \\
\hline TI & 9 & 41,52 & 33,44 & $42,92^{\mathrm{a}}$ & 11,15 \\
TM & 28 & 47,02 & 33,32 & $33,62^{\text {ab }}$ & 6,30 \\
TF & 30 & 53,56 & 28,43 & $48,66^{\text {ac }}$ & 5,19 \\
MP & 55 & 29,06 & 23,30 & $21,76^{\text {abd }}$ & 3,14
\end{tabular}

Letras diferentes na mesma coluna indicam haver diferença significativa. Valor de $\mathrm{p}<0,008$.

e, por conseguinte, estabelecer o nível de estresse que esse produto irá enfrentar na hora do parto (Vaala 2002).

A dificuldade na obtenção dos fluidos fetais equinos com segurança é um fator limitante do conhecimento da fisiologia obstétrica nesta espécie, fato que não ocorreu com outras espécies como a humana, pequenos ruminantes e até mesmo bovina. A amniocentecentese em equinos desencadeou abortamentos por infecções geradas pelas diversas perfurações para obtenção dos fluidos fetais durante muitos anos (Ausbury \& Leblanc 1993). No entanto, o advento do ultrassom permitiu a o acompanhamento do cateter de coleta em laparocenteses transabdominais trazendo avanços para o procedimento (Schimidt et al.1991). Não obstante, o custo elevado deste material e procedimento inviabiliza este procedimento na rotina dos hospitais veterinários (Zanella 2008). Por este motivo, optou-se por trabalhar com peças oriundas de frigoríficos comerciais e de colheitas realizadas durante o parto natural para diminuir o custo financeiro deste experimento.

Foram colhidas 67 amostras de peças de frigoríficos comerciais e 55 amostras durante partos naturais. 0 número de amostras obtidas nos frigoríficos, principalmente do grupo TI, foi limitado pela distância entre os frigoríficos localizados na cidade de São Gabriel/RS $\left(30^{\circ} 46^{\circ} 42^{\prime \prime}\right.$ S, $54^{\circ} 52^{\prime} 50^{\prime \prime}$ ) $)$ e Araguari/MG (18 $\left.39^{\circ} 4^{\prime \prime} \mathrm{S}, 48^{\circ} 11^{\prime} 7^{\prime \prime} 0\right)$ e o laboratório onde as amostras foram analisadas (Botucatu/ SP - $22^{\circ} 53^{\prime} 09^{\prime \prime} S, 48^{\circ} 26^{\prime} 42^{\prime \prime}$ ), e ainda, do número restrito de éguas prenhes abatidas.

No presente experimento, os valores das medianas encontrados para a variável $\mathrm{pH}$, não demonstraram diferenças entre os grupos estudados. Este dado não surpreende e demonstra a capacidade do organismo equino em manter o equilíbrio fisiológico dos fluídos fetais durante a gestação em compatibilidade com o desenvolvimento do feto. No en- 

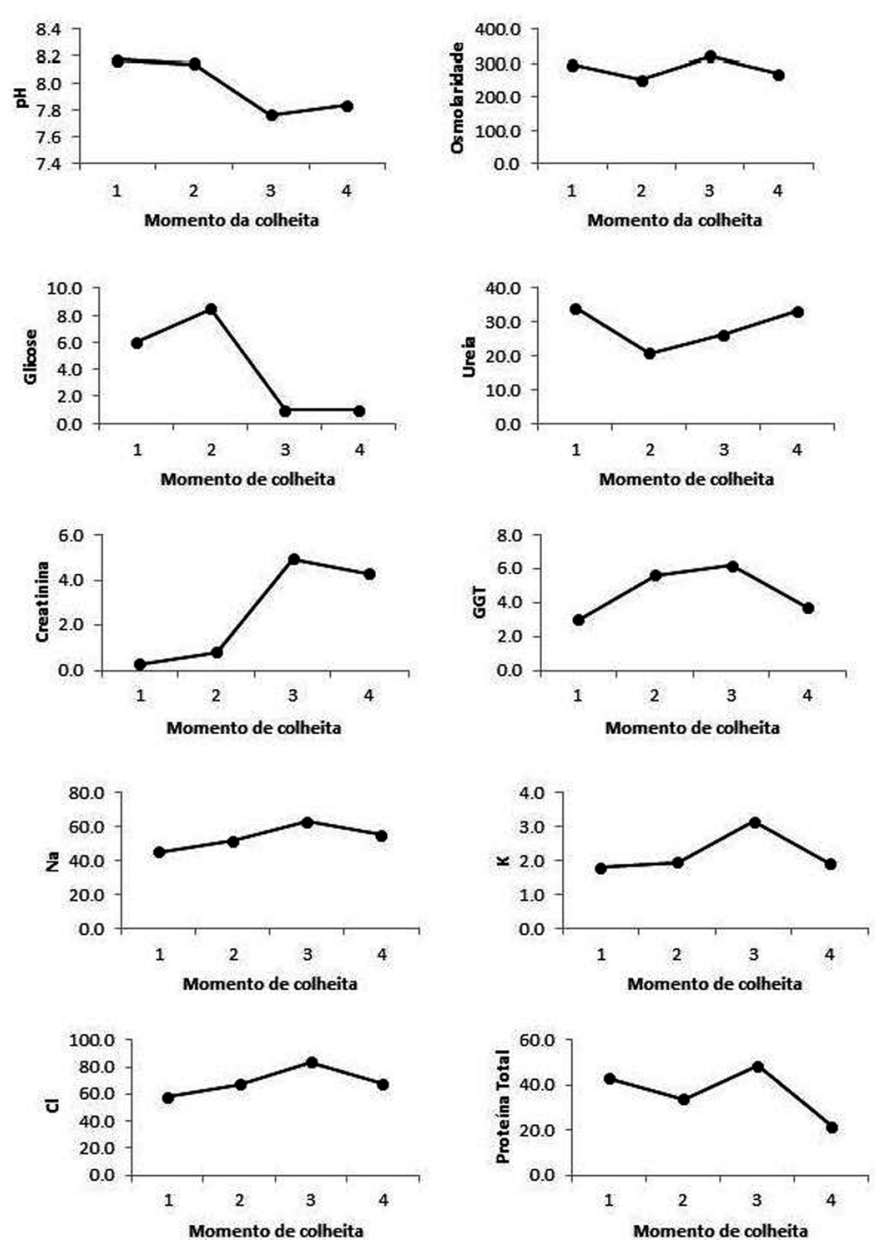

Fig.1. Medianas dos valores encontrados para $\mathrm{pH}$, osmolaridade (mOsm/L), glicose $(\mathrm{mg} / \mathrm{dL})$, uréia $(\mathrm{mg} / \mathrm{dL})$, creatinina $(\mathrm{mg} /$ $\mathrm{dL})$, gamma-GT (IU/L), sódium $(\mathrm{mEq} / \mathrm{L})$, potássio $(\mathrm{mEq} / \mathrm{L})$, cloretos $(\mathrm{mEq} / \mathrm{L})$ e proteína total $(\mathrm{mg} / \mathrm{dL})$ no líquido amniótico equino colhido em diferentes momentos: $1=$ terço inicial, $2=$ terço médio, 3 = terço final da gestação, 4 = momento do parto.

tanto os valores médios do $\mathrm{pH}$ do líquido amniótico ovino foram significativamente menores nos terços médio e final da gestação de ovelhas em relação ao terço inicial (Prestes et al., 2001), porém este fato pode estar relacionado com uma manutenção da atividade enzimática nas amostras após a colheita, alterando os constituintes e consequentemente o valor do $\mathrm{pH}$ (McDougall 1949).

Para a osmolaridade os valores das medianas encontradas foram TI:296,5; TM:250,0; TF: 320,0 e MP: 269,0 (mOsm/L) e não diferiram significativamente nos grupos estudados. Este fato não foi observado por Prestes et al. (2001) que relataram que a osmolaridade dos terços inicial e médio foi significativamente mais elevada do que a encontrada no terço final da gestação de ovelhas. No entanto, Mellor e Slater (1974) afirmaram que a entrada de urina pode reduzir a osmolaridade do líquido amniótico (Alexander et al. 1958).

A mediana dos valores encontrados em $\mathrm{mg} / \mathrm{dL}$ para glicose não diferiram entre os grupos TI $\left(6,0^{\mathrm{a}}\right)$ e TM $\left(8,5^{\mathrm{a}}\right)$ e entre os grupos TF e MP $\left(1,0^{\mathrm{b}} ; 1,0^{\mathrm{b}}\right.$ respectivamente), no entanto os valores encontrados na fase final da gestação e momento do parto foram significativamente menores do que os encontrados nas fases inicial e média. Este fato corrobora com os resultados encontrados por Zanella (2008), que relatou uma concentração significativamente menor de glicose no líquido amniótico colhido na fase final da gestação em comparação às fases inicial e média, e com os relatos de Prestes et al. (2001), que demonstraram uma redução nos valores de glicose ao longo do desenvolvimento da gestação em ovinos. Este fato deve-se provavelmente a ingestão de glicose pelo feto a partir do início do reflexo de deglutição fetal.

As medianas das concentrações de uréia não diferiram estatisticamente entre os grupos estudados, estando de acordo com o resultado encontrado por Barreto et al. (2006). Porém quando avaliada a hipótese de que pelo menos o valor de um grupo foi diferente do outro, observou-se um valor de p de 0,05174, indicando uma tendência estatística para essa diferença. Outro experimento com líquido amniótico equino e um experimento com líquido amniótico ovino demonstraram que a média de concentração de uréia foi significativamente maior na fase inicial da gestação (Prestes et al. 2001, Zanella 2008,). No entanto, esta diferença pode não ter sido significativa devido ao baixo número de amostras de fase inicial de gestação no presente experimento. Os valores das médias de concentração de uréia encontrados no momento do parto $(36,54 \pm 16,35 \mathrm{mg} /$ dL) foram semelhantes aos descritos por Kochhar et al. (1997). A diminuição da concentração da uréia no líquido amniótico e o aumento da concentração da uréia no líquido alantoideano durante o desenvolvimento da gestação demonstrado por Zanella (2008) pode ter relação com o desenvolvimento da atividade renal do feto.

As medianas das concentrações de creatinina (mg/ dL) demonstraram um padrão significativamente crescente durante as fases gestacionais $\mathrm{TI}, \mathrm{TM}$ e TF $\left(0,30^{\mathrm{a}} ; 0,80^{\mathrm{b}}\right.$; $4,95^{\mathrm{C}}$ respectivamente) e não diferiram entre a fase final e momento do parto (TF: $4,95^{c}$; $4,90^{c}$ ), corroborando com os resultados encontrados por Prestes et al (2006) em ovinos e Zanella (2008) em equinos e está em desacordo com o relatado por Barreto et al (2006) em cães. Este aumento foi ainda significativamente maior no líquido alantoideano de equinos (Zanella 2008). Segundo Prestes et al. (2001) o aumento da taxa de creatinina está correlacionado com a maturidade renal do feto, e também; com a atividade muscular fetal (Kochhar et al. 1997).

Quanto a Gama GT, os valores das medianas diferiram apenas entre os grupos TF e MP $\left(6,20^{\text {ab }} \mathrm{U} / \mathrm{L} ; 3,70^{\text {ac }} \mathrm{UI} / \mathrm{L}\right.$ respectivamente). Esta enzima está presente nos canais de secreção e excreção e seu papel no líquido amniótico não está claramente definido. Sabe-se que desempenha importante papel na regulação do GSH (Tripeptídeo Glutationa) intracelular, com função antioxidante, catalisando a transferência do grupo Gama Glutamil para outros peptídeos, aminoácidos e água que são facilmente utilizáveis pela célula. É encontrada em concentrações mais altas nos rins, pâncreas e fígado, participando também no metabolismo de mediadores de várias funções fisiológicas como leucotrienos e prostaglandinas. Apenas Williams et al. (1993), encontraram em éguas, concentração de Gama GT mais alta no líquido alantoideano que no amniótico. 
As medianas das concentrações dos íons sódio e cloreto não diferiram significativamente entre os grupos estudados, concordando com os resultados demonstrados por Barreto et al. (2006) em cadelas. Porém, discorda com os resultados encontrados por Prestes et al. (2001), que apresentaram valores de $\mathrm{Cl}$ significativamente diferentes entre as fases da gestação de ovinos e concentração de Na significativamente decrescente durante as fases gestacionais; e Zanella (2008) que apresentou taxas de $\mathrm{Cl}$ e $\mathrm{Na}$ mais elevadas no segundo terço da gestação. Quanto aos valores do íon potássio, encontramos diferença significativa apenas entre os grupos TF e MP $\left(3,15^{\mathrm{ab}} ; 1,90^{\mathrm{ac}}\right)$, também discordando dos resultados apresentados por Zanella et al. (2007). $O$ valor mais elevado do íon K no líquido alontoideano do que no líquido amniótico relatado na literatura, está relacionado com o aumento de produção de urina pelo feto e bem como pelo aumento da atividade metabólica do mesmo (Kochhar et al. 1997, Prestes et al. 2001), no entanto não realizamos tal comparação neste estudo.

As medianas das concentrações de proteína total diferiram entre os grupos TM e TF $\left(33,62^{\text {ab }} ; 48,62^{\text {ac }}\right)$ e TF e MP $\left(48,66^{\text {ac; }} ; 21,76^{\text {abd }}\right)$. Esta diferença significativa apresentou um padrão heterogêneo de concentração de proteína total que está em desacordo com os resultados apresentados por Zanella (2008) que demonstrou um aumento significativo na concentração de PT no terço médio da gestação em relação aos grupos terço inicial e terço final, enquanto que Prestes et al. (2001) apresentaram uma elevação significativa na concentração no terço médio da gestação de ovinos que manteve-se elevada no terço final da gestação. Não obstante, os valores das concentrações de PT encontradas neste experimento corroboram com experimentos realizados em outras espécies no que tange a pequena quantidade de proteína presente no líquido amniótico (Reddy et al. 1995, Prestes et al. 2001, Barreto et al. 2006, Zanella et al. 2008). Pois, neste experimento, foi necessária a utilização de um Kit de proteínas de baixa concentração que é capaz de detectar quantidades mínimas de proteína, já que o Kit utilizado na rotina não foi sensível o bastante para dosar a proteína existente no líquido amniótico equino.

\section{CONCLUSÕES}

Podemos concluir que os valores encontrados na avaliação bioquímica dos grupos estudados e suas comparações variam em relação aos escassos e antigos estudos realizados para a espécie equina, demonstrando a necessidade de realização de mais trabalhos para o estabelecimento do perfil bioquímico do líquido amniótico equino colhido em diferentes fases gestacionais e momento do parto visando, no futuro, a utilização destes parâmetros na avaliação da maturidade e viabilidade fetal equina.

Agradecimentos.- Às agências FAPESP, Fundunesp e CAPES pelo apoio financeiro; aos Frigoríficos Pomar (Araguari, MG) e Foresta (São Gabriel, RS) e à Universidade Federal de Pelotas pela colaboração na execução desta pesquisa. Ao Professor José C.F. Pantoja pela assessoria quanto à análise estatística e ao médico veterinário Tiago Martins pelo auxílio na colheita de amostras no Frigorífico Pomar.

\section{REFERÊNCIAS}

Alexander D.P., Nixon D.A., Widdas W.F. \& Wohlzogen F.X. 1958. Gestation variations in the composition of the foetal fluids and foetal urine in the sheep. J. Physiol.140:1-13.

Ausbury A.C. \& Leblanc M.M. 1993. The placenta, p.509-516. In: McKinnon A.O. (Ed.), Equine Reproduction. $3^{\text {rd }}$ ed. Lea ad Febiger, Pensylvania.

Barreto C.S., Souza F.F. \& Prestes N.C. 2006. Avaliação bioquímica dos fluidos amniótico e alantoideano de cadelas dentre 30 e 40 dias de gestação. Revta Port. Ciênc. Vet. 101:215-217.

Çagdas A., Aydinli K., Irez T., Temizyrek K. \& Apak M. 2000. Evaluation of the fetal kidneymaturation by assessment of amniotic fluid alph-1 microglobulin levels. Eur. J. Obstet. Gyncol. Reprod. Biol. 90:55-61.

Campana S.G., Chávez J.H. \& Haas P. 2003. Diagnóstico laboratorial do líquido fetal. J. Bras. Patol. Med. Lab. 39:215-218.

Jonker F.H. 2004. Fetal death: comparative in large domestical animals. Ann. Repr. Sci. 82:415-430.

Kjeldsberg C. \& Knight J. 1993. Body fluids: laboratory examination of amniotic, cerebrospinal, serous and synovial fluids. $3^{\text {rd }}$ ed. American Society of Chemical Pathologists, Chicago. 436p.

Kleinbaum D.G., Kupper L.L., Nizam A. \& Muller K.E. 2007. Applied Regression Analysis and Multivariable Methods. Duxbury Press, Belmont. 906p.

Kochhar H.P.S., Simram P.S., Nanda A.S. \& Kaur R. 1997. Comparative biochemical indices of fetal fluids in normal foaling and stressful delivery in Indian Thoroughbred. J. Equine Vet. Sci. 17(4):206-210.

Martins L.R., Prestes N.C. \& Lopes M.D. 2004. Análise comparativa dos componentes bioquímicos no líquido amniótico de fetos e soro de cadelas (Cannis familiaris). Ars Vet. 20(1):115-122.

McDougall E.I. 1949. The composition of foetal fluids of sheep at different stages of gestation. Biochem. J. 45:397-400.

Moore K. \& Persaud T.V.N. 2000. Placenta e membranas fetais, p.123160. In: Moore K. (Ed.), Embriologia Clínica. 6aㅡ ed. Guanabara, Rio de Janeiro.

Naves C.S., Vieira R.C., Diniz E.G., Jacomini J.O., Belleti M.E. \& Oliveira R. 2008. Desenvolvimento morfológico dos ovários em fetos equinos sem raça definida. Ciência Rural 38(2):416-422.

Pagano M. \& Gauvreau K. 2000. Principles of Biostatistics. Duxbury Press, Belmont, California. 592p.

Prestes N.C., Chalhoub M.D., Lopes M.D. \& Takahira R.K. 2001. Amniocentesis and biochemical evaluation of amniotic fluid in ewes at 70,100 e 145 days of pregnancy. Small Rumin. Res. 39:277-281.

Reddy A.P., Reddy V.S.C., Rao A.S., Sharma G.P., Reddy G.V.N., Reddy T.J. \& Reddy V.S. 1995. Biochemical studies in the ewe (Ovis aries) amniotic fluid during different phases of gestation. Indian J. Anim. Sci. 10:321323.

Schmidt A.R., Williams M.A., Carleton C.L., Darien B.J. \& Derksen F.J. 1991. Evaluation of transabdominal ultrasound-guided amniocentesis in the late gestational mare. Equine Vet. J. 23(4):261-265.

Souza H.E.M., Cardoso E.C., Barnabé V.H. \& Vale W.G. 1999. Alterações bioquímicas dos líquidos fetais de bubalinos com avanço da gestação. Revta Bras. Reprod. Anim. 23:302-304.

Vaala W. \& House J. 2002. Manifestation disease in the neonate, p.319-381. In: Smith B.S. (Ed.), Large Animal Internal Medicine. $3^{\text {rd }}$ ed. Mosby, St Louis.

Williams A., Wallace S., Tyler J., McCall C., Gutierrez A. \& Spano J.S. 1993. Biochemical characteristics of amniotic and allantoic fluid in late gestational mares. Theriogenology 40(6):1251-1257.

Zanella L.F. 2008. Análise bioquímica do líquido amniótico e alantoideano de Equus caballus em diferentes fases da gestação. Tese de Doutorado em Medicina Veterinária, Faculdade de Medicina Veterinária e Zootecnia, Universidade Estadual Paulista, Botucatu, SP. 90p. 